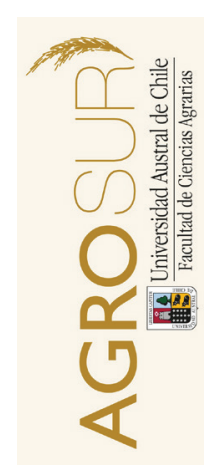

\section{Pre-compactación como herramienta para mejorar la relación agua/aire en habilitación de suelos post-extracción de áridos}

\author{
Pre-compaction as tool to improve water/air relationship in soil \\ reclamation after subsurface sand strata extraction
}

\author{
Seguel, 0. ${ }^{a *}$, Cárcamo, I. ${ }^{a}$, Casanova, M. $^{a}$, Homer, I. ${ }^{a}$, Haberland, J. ${ }^{a}$, Benavides, C. ${ }^{a}$ \\ ${ }^{a}$ Universidad de Chile, Facultad de Ciencias Agronómicas, Departamento de Ingeniería y Suelos. \\ Casilla 1004, Santiago, Chile.
}

\begin{abstract}
A B S T R A C T
In order to reclaim a disturbed Inceptisol (1 m depth, sandy loam) after subsurface sand extraction, pre-compaction labours with different water contents were made, to assess the impact of this management on some soil physical properties. Randomized field plots $\left(20 \mathrm{~m}^{2}\right)$ were used to achieve three increasing pre-compaction levels (T1, T2 and T3) and a control without compaction (T0), considering four replicates. A pea crop (Pisum sativum L.) was established as biological indicator. Bulk density, water content, pre-compaction by Proctor test (laboratory and field), infiltration rate and resistance to penetration were measured in the soil, while dry matter was measured in the crop. Soil bulk density values ranged between $1.25 \mathrm{Mg} \mathrm{m}^{-3}$ for $\mathrm{T} 0$ and $1.49 \mathrm{Mg} \mathrm{m}^{-3}$ for T3, the higher precompaction treatment. Field Proctor test showed results similar to those found in the laboratory assessment, with a parabolic adjustment pattern. Soil resistance to penetration reached maximum values fluctuating between 180 and $280 \mathrm{kPa}$, concentrating in the surface and dissipating below $30 \mathrm{~cm}$ depth. Increased water storage was generated by pre-compaction treatments, although T3 promoted a significantly lower infiltration rate. The best crop yield was observed in T2, with a bulk density of $1.41 \mathrm{Mg} \mathrm{m}^{-3}$, showing the best water/air/ resistance relation which resulted in a higher crop yield.
\end{abstract}

\title{
RESUMEN
}

Con el objeto de recuperar un suelo agrícola franco arenoso disturbado por la extracción de estratas de arenas subsuperficiales, se realizó una labor de pre-compactación con distinto contenido de agua, para evaluar el efecto sobre algunas propiedades físicas del suelo. Se utilizaron parcelas de $20 \mathrm{~m}^{2}$ distribuidas al azar, dentro de las cuales se lograron generar tres niveles de pre-compactación con rodillo (T1, T2 y T3), más un testigo (T0) sin compactar, todas con cuatro repeticiones. Se utilizó un cultivo de arveja (Pisum sativum L.) como indicador. En el suelo se midió la densidad aparente, el contenido de agua, la pre-compactación con el Test de Próctor, la velocidad de infiltración y la resistencia a la penetración; en el cultivo se midió el rendimiento en materia seca. Los valores de densidad aparente variaron entre $1,25 \mathrm{Mg} \mathrm{m}^{-3}$ para el testigo (T0) y 1,49 Mg $\mathrm{m}^{-3}$ para el tratamiento de mayor compactación (T3). La pre-compactación en campo presentó un comportamiento similar al obtenido con el Test de Próctor, con un ajuste parabólico. La resistencia a la penetración alcanzó valores máximos que fluctuaron entre los 180 y $280 \mathrm{kPa}$, concentrándose en los $30 \mathrm{~cm}$ superficiales y disipándose en profundidad. Los tratamientos de pre-compactación generaron un aumento en el almacenamiento de agua, aunque en T3 generó la menor tasa de infiltración. La mejor condición se alcanzó en el tratamiento T2, con una densidad aparente de 1,41 Mg m³ , logrando la mejor condición en la relación agua/aire/resistencia, traduciéndose en los mayores rendimientos de materia seca.

Palabras clave: Pre-compactación, Test de Próctor, densidad aparente, porosidad, resistencia mecánica.

\section{INTRODUCCIÓN}

La extracción de áridos es una actividad que ejerce presión sobre los recursos naturales. Un caso especialmente importante es la efectuada en terrenos de uso agrícola, donde los materiales de interés se encuentran en un horizonte subsuperficial y que para su extracción, se necesita remover las capas más superficiales del suelo. Este manejo altera las características desarrolladas durante el proceso de pedogénesis, tales como estructu- ra, densidad aparente, capacidad de retención de agua, propiedades de transmisión hidráulica, entre otras. La alteración de estas propiedades, del punto de vista agrícola, hace perder la adecuada funcionalidad del suelo como un medio físico para el arraigamiento de los cultivos (Macaya y Gallardo, 2007; Horn y Fleige, 2009).

La capacidad de penetración de las raíces y el arraigamiento de los cultivos dependen de la porosidad y de la resistencia mecánica. Estas propiedades edáficas muestran una variación espacial asociada a la estruc- 
tura del suelo, de manera que las raíces se concentran en las zonas del perfil con resistencia a la penetración baja y con una relación aire/agua favorable (Ellies et al., 1992; Silva et al., 2011).

Los cultivos necesitan en promedio un $10 \%$ de porosidad mayor a $50 \mu \mathrm{m}$, tamaño de poros que asegura el movimiento de agua e intercambio gaseoso rápido (Pagliai y Vignozzi, 2002). Sin embargo, una macroporosidad excesiva puede ser contraproducente en sedimentos frescos o en suelos cuya estructura ha sido destruida, como es el caso de la extracción de materiales subsuperficiales. En esta condición, disminuye la productividad agrícola al reiniciarse la utilización del suelo (re-cultivación), por cuanto se pierde la capacidad de soporte para tránsito agrícola (Hartge, 1988). Se genera además una infiltración y percolación de agua excesivamente rápidas (Pagliai y Vignozzi, 2002) y un efecto de dilución de la materia orgánica y los nutrientes del suelo (Macaya y Gallardo, 2007). En suelos de texturas medias a gruesas, una opción para la re-cultivación, hasta que el suelo se re-estructure en forma natural o con aportes de materia orgánica, la constituye una labor de pre-compactación, de manera de asegurar un equilibrio entre las fases líquida, sólida y gaseosa, permitiendo un tráfico sustentable de la maquinaria agrícola y un desarrollo radical no limitante.

La pre-compactación de suelos es una práctica correspondiente a una labor de asentamiento o re-arreglo de las partículas y/o agregados, a través de una carga mecánica en superficie (tensiones externas), sin incurrir en un aumento excesivo de la resistencia mecánica del suelo (Hartge, 1988). Por otra parte, una excesiva compactación provoca un aumento en la densidad aparente, además de una reducción del espacio poroso, cambiando la continuidad de este último, ya que se modifica la orientación de los macroporos, desde un patrón vertical a otro horizontal (Ellies et al., 1992; Horn, 2003).

Una labor de pre-compactación en suelos excesivamente sueltos provoca una disminución de la macroporosidad $(>50 \mu \mathrm{m})$, con un aumento de poros de almacenamiento de agua (Lipiec, 2004). Junto con ello, en el rango ideal de potencial mátrico de los cultivos agrícolas (> - $100 \mathrm{kPa}$ ), aumenta la conductividad hidráulica (Horn, 2003). En sectores con pendiente, la pre-compactación aumenta el escurrimiento y el riesgo de erosión, pero en suelos arenosos muy permeables podría disminuir la lixiviación de nutrientes (Lipiec, 2004).

Una labor de pre-compactación adecuada favorece la relación de fases para el desarrollo de la biomasa vegetal, lo que se traduce en un aumento de la producción (Lipiec, 2004; Medvedev, 2004). Además, con esta labor se acelera el asentamiento, lo que incrementa la capacidad de soporte del suelo posterior a la disturbación. Así, es posible definir un asentamiento óptimo, como aquel grado de pre-compactación en el que se logra la mejor relación entre sustentabilidad mecánica y funcionalidad del sistema poroso (Horn et al., 2007).

Esta investigación plantea que las labores de precompactación y la relación existente entre el contenido de agua y el aumento de la densidad aparente del suelo generan un nivel intermedio de asentamiento (óptimo), que favorecerá la relación entre los contenidos de agua, aire y resistencia mecánica para un determinado cultivo. En consecuencia, el objetivo principal de esta investigación fue evaluar el efecto de distintos niveles de precompactación sobre propiedades físicas de un suelo franco arenoso re-cultivado post-extracción de áridos.

\section{MATERIALES Y MÉTODOS}

\section{Materiales}

El estudio se realizó en Santiago, Comuna de Maipú, dentro de la Estación Experimental Germán Greve Silva de la Universidad de Chile ( $33^{\circ} 28^{\prime}$ LS- $70^{\circ} 50^{\prime}$ LO) entre marzo y noviembre del año 2006 . El sector posee un clima templado cálido, con lluvias que presentan un monto interanual muy variable, con un promedio de $317 \mathrm{~mm}$, concentrado en los meses de invierno y con un periodo seco que puede alcanzar hasta 10 meses (Olivares et al., 1998). Los análisis de laboratorio fueron realizados en dependencias del Departamento de Ingeniería y Suelos, en los laboratorios de Física de Suelos y de Química de Suelos y Aguas de la Facultad de Ciencias Agronómicas de la Universidad de Chile.

Se trabajó en un sector dedicado a la extracción de áridos con un material disturbado que, previo a la extracción, correspondía a un Inceptisol de la Serie Rinconada de Lo Vial (Familia franca, gruesa, mixta, térmica de los Fluventic Haploxerepts) (CIREN, 1996). Este suelo es de origen aluvial y estratificado, moderadamente profundo, con un solum que varía entre 63 y $107 \mathrm{~cm}$. Se presenta en posición de terrazas planas, con o sin microrelieve. La clase textural es franco arenosa a franco arenosa muy fina y ocasionalmente areno francosa; presenta un fragipán a los $100 \mathrm{~cm}$ en promedio, desarrollado sobre clases texturales arenosas o areno francosas donde no penetran las raíces. El drenaje del suelo varía de bueno a imperfecto, presentando nivel freático bajo los $120 \mathrm{~cm}$; el horizonte superficial del suelo no disturbado presenta un contenido de materia orgánica de 2,9\% y una conductividad eléctrica de $2,1 \mathrm{dS} \mathrm{m}^{-1}$, los cuales disminuyen en profundidad (CIREN, 1996).

\section{Método}

\section{Proceso de extracción de áridos}

Las faenas de extracción de áridos se realizaron en febrero de 2006, concentrándose bajo los primeros 
$100 \mathrm{~cm}$ del suelo en estratas de arena de 2 a $4 \mathrm{~m}$ de espesor. Para esto, el suelo es removido y apilado con una retroexcavadora en fajas alrededor del sitio de extracción (Figura 1).

Posterior a la remoción de la capa superficial, la estrata de arena es sacada (se extraen en promedio $2 \mathrm{~m}$ de espesor), para luego restituir mecánicamente el suelo disturbado sobre el piso dejado por la extracción. Como consecuencia, el sitio ve reducida su cota en $2 \mathrm{~m}$ y queda un material mezclado, con un microrelieve distinto al que tenía originalmente. Este hecho imposibilita mantener el manejo previo a la extracción, ya que el suelo queda suelto y carente de soporte para el tránsito de maquinaria agrícola; además, el nivel freático alcanza casi la superficie, lo que dificulta aún más su rehabilitación.

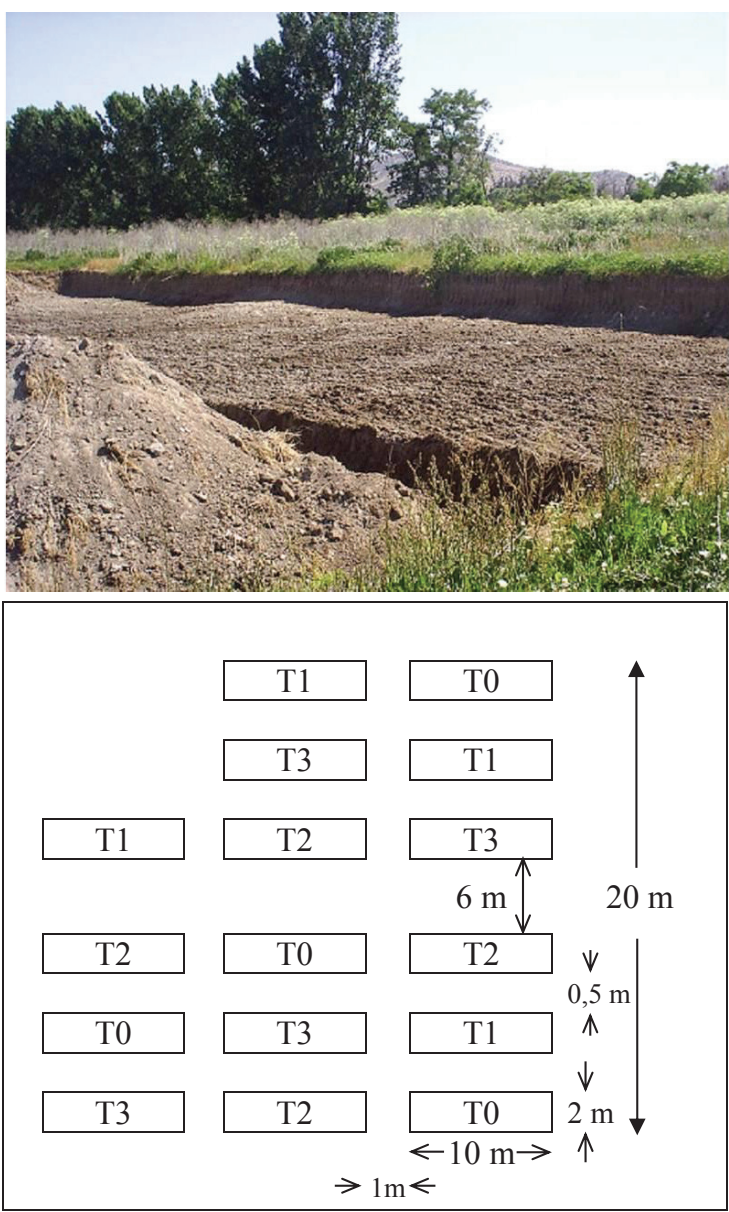

Figura 1: Sitio de la extracción de arena. Arriba (en el primer plano a la izquierda), depósito de suelo dejando al descubierto la estrata de arena; abajo, distribución de los tratamientos en el ensayo.

Figure 1: Sand extraction-site. Above (in the foreground to the left), soil deposit exposing the sandy strata; below, distribution of the treatments in the trial.

\section{Establecimiento del ensayo}

Sobre el material suelo disturbado y repuesto posterior a la extracción de arena subsuperficial, se establecieron 16 parcelas o unidades experimentales de $10 \mathrm{~m}$ de largo y $2 \mathrm{~m}$ de ancho (Figura 1). Mediante el uso de un rodillo compactador $(200 \mathrm{~kg}$ de masa y $1 \mathrm{~m}$ de ancho) transitado a una velocidad de $7 \mathrm{~km} \mathrm{~h}^{-1}$, se aplicaron niveles de pre-compactación con distintos contenidos de agua (distintos tiempos posteriores a un evento de lluvia artificial), luego de lo cual se estableció un cultivo de arveja (P. sativum), el cual se seleccionó por su rapidez de crecimiento y capacidad de fijación de $\mathrm{N}$ atmosférico.

Los tratamientos de pre-compactación, descritos en el Cuadro 1, se distribuyeron al azar en las unidades experimentales, considerando cuatro repeticiones por tratamiento.

Para establecer el momento óptimo de compactación $\left(t_{i}\right)$ se empleó un criterio en base a una curva de desecamiento. Ésta fue realizada en un cilindro de PVC de $200 \mathrm{~mm}$ de diámetro y $600 \mathrm{~mm}$ de altura, con la cual se determinó el contenido de agua a distintos intervalos de tiempo con posterioridad a un riego simulado de $40 \mathrm{~mm}$.

La siembra se realizó en forma manual en Agosto del 2006, para lo cual se utilizaron implementos para surcar y disponer tanto la semilla de arveja como el fertilizante. La fertilización del cultivo se basó en un análisis de suelo completo, de manera de satisfacer sus necesidades. Las dosis aplicadas a la siembra por hectárea fueron de 30 unidades de $\mathrm{N}$ (salitre potásico) y 150 unidades de $\mathrm{P}_{2} \mathrm{O}_{5}$ (súper fosfato triple). Todas las labores de establecimiento y un control periódico de malezas fueron realizadas en forma manual para no generar esfuerzos extras ajenos al suelo del experimento. Se estableció un sistema de riego por aspersión móvil, realizando un balance hídrico con la información climática de la zona para estimar los requerimientos de

Cuadro 1: Tratamientos de pre-compactación.

Table 1: Pre-compaction treatments.

Tratamiento Condición

T0 Testigo, suelo sin compactar (post extracción) Suelo pre-compactado con 1 pasada de

$\mathrm{T} 1$ rodillo al tiempo $\mathrm{t}_{1}$ después de un riego por aspersión. rodillo al tiempo $t_{2}$ después de un riego por aspersión.

Suelo pre-compactado con 1 pasada de

T3 rodillo al tiempo $t_{3}$ después de un riego por aspersión. 
agua. Se utilizaron los coeficientes de cultivos de acuerdo a Allen et al. (2006), a saber, 0,5 en el establecimiento, 1,15 en etapa media y 0,3 en la etapa final, más la evapotranspiración potencial cuantificada mediante una bandeja clase A estándar ubicada en la estación meteorológica de la Estación Experimental. El tiempo de riego se determinó para suplir el 100\% de la demanda hídrica del cultivo, con una frecuencia de 3 a 5 días, totalizando en la temporada un equivalente de 2.800 $\mathrm{m}^{3} \mathrm{ha}^{-1}$.

\section{Evaluaciones}

Al cabo de un mes de establecido el ensayo, una vez que se logró el asentamiento final del suelo como consecuencia de los riegos, se tomaron muestras no disturbadas a dos profundidades (0-20, 20-30 cm), una muestra por unidad experimental ( 4 tratamientos, 2 profundidades, 4 repeticiones, $n=32$ ), con cilindros metálicos de $50 \mathrm{~mm}$ de altura y $59 \mathrm{~mm}$ de diámetro (136,7 $\mathrm{cm}^{3}$ ) (Grossman y Reinsch, 2002). En ellas se determinó la densidad aparente (Da) alcanzada con los tratamientos de pre-compactación y el contenido gravimétrico de agua. La densidad real (Dr) se midió por el método del picnómetro (Flint y Flint, 2002), con lo que se determinó el número poroso $(e)$ mediante la relación $e=\mathrm{Dr} / \mathrm{Da}-1$, equivalente a volumen de poros/ volumen de sólidos.

La información obtenida de terreno (Da y contenido de agua, que corresponde al Próctor de campo) se comparó con la curva obtenida a través del Test de Próctor (Kézdi, 1980), realizada con el material alterado completo y con la fracción $<2 \mathrm{~mm}$. El Test de Próctor usa un criterio estándar aceptado universalmente según la American Society for Testing and Materials (ASTM, 1995) y ha sido diseñado para determinar el contenido de agua óptimo del suelo, al cual la compactación logra su mayor eficiencia de energía.

A partir de la curva de retención de agua, determinada de acuerdo a Dane y Hopmans (2002) con muestras no disturbadas, se obtuvo la distribución de poros por tamaño, relacionando su diámetro con la energía de retención. Esto permitió clasificar los poros en poros de drenaje rápido ( $\mathrm{PDR},>50 \mu \mathrm{m}$, retención entre saturación y -6 kPa), poros de drenaje lento (PDL, 10$50 \mu \mathrm{m}$, retención entre -6 y $-33 \mathrm{kPa}$ ), poros de agua útil (PAU, 0,2-10 $\mu \mathrm{m}$, retención entre -33 y -1500 kPa) y poros de agua inútil (PAI, $<0,2 \mu \mathrm{m}$, retención a -1500 $\mathrm{kPa}$ ).

Al final del ensayo, previo a la cosecha del cultivo y cuando la raíz de éste ya había ejercido su efecto sobre el suelo, se estimó la conductividad hidráulica a partir de la Velocidad de Infiltración estabilizada medida con cilindro infiltrómetro (Reynolds et al., 2002). Se determinó además la resistencia a la penetración mediante un penetrómetro de cono (Koolen y Kuipers, 1983), en un rango de medición de 0 a $400 \mathrm{kPa}$, a las profundidades de 5, 15, 25, 35, 55 y $75 \mathrm{~cm}$, en tres sitios por unidad experimental.

Finalmente se evaluó el cultivo establecido en términos de la producción de materia seca, cosechando $1 \mathrm{~m}^{2}$ de material con raíces por unidad experimental, secando el material fresco en estufa $\left(70^{\circ} \mathrm{C}\right)$ hasta alcanzar masa constante.

\section{Diseño y análisis estadístico}

El diseño estadístico correspondió a parcelas distribuidas al azar. Como los tratamientos generaron distintos niveles de pre-compactación, los datos obtenidos fueron analizados en términos de comparaciones entre tratamientos, para cada una de las propiedades estudiadas, por la vía de un análisis de varianza ( $\mathrm{p} \leq 0,05)$. Cuando existieron diferencias estadísticamente significativas se realizó la prueba de rango múltiple (LSD, $\alpha$ $\leq 0,05)$. Para la infiltración acumulada estabilizada en el tiempo, se realizó una prueba t de comparación de pendientes. Finalmente, cuando el análisis de la información lo requirió, se realizaron ajustes de regresión entre variables, evaluando el coeficiente de determinación con un nivel de significancia de 95\%.

\section{RESULTADOS Y DISCUSIÓN}

Con posterioridad a la extracción de áridos, se apreció una pérdida de estructura y una mezcla de los horizontes originales, con presencia de trozos de fragipán distribuidos aleatoriamente. El perfil presentó como consecuencia cierta homogeneidad de los contenidos de nutrientes y de materia orgánica (MO), generándose disminuciones de ambos por efecto de dilución, es decir, una disminución de la fertilidad natural del suelo.

El análisis de suelo post extracción de áridos indicó un valor de $\mathrm{pH}$ de 7,23 y un contenido de MO de 0,55\%; por otra parte, los macronutrientes se presentaron en niveles muy bajos para $\mathrm{N}$ y $\mathrm{P}$, con valores de $9 \mathrm{mg} \mathrm{kg}^{-1}$ de $\mathrm{N}$ y $6 \mathrm{mg} \mathrm{kg}^{-1}$ de $\mathrm{P}_{2} \mathrm{O}_{5}$. Los contenidos de potasio, sin embargo, permanecieron altos ( $224 \mathrm{mg} \mathrm{kg}^{-1} \mathrm{de}_{2} \mathrm{O}$ ), lo que justificó que la fertilización del cultivo se centrara en suplir las necesidades referidas a nitrógeno y fósforo. Por otra parte, la cercanía al nivel freático provocó aumentos de la conductividad eléctrica (CE), alcanzando niveles de 6,87 $\mathrm{dS} \mathrm{m}^{-1}$ por efecto del ascenso capilar y evaporación de agua en la superficie del suelo.

\section{Comportamiento mecánico del suelo}

La curva de agotamiento de agua, empleada para la pre-compactación, se aprecia en la Figura 2 considerando distintos contenidos de agua del suelo en el tiempo. Al inicio (tiempo $=0$ ) se aplicó una lámina de riego de $40 \mathrm{~mm}$. 


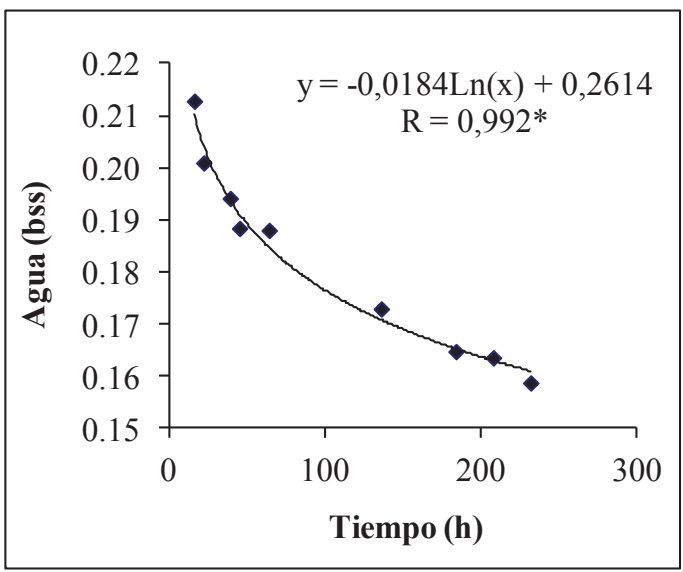

Figura 2: Variación del contenido de agua en el tiempo del material de reposición posterior a la extracción de arenas. * Significativo al 95\%.

Figure 2: Water content variation over time of the replenishment material after extraction of sand. * Significant at $95 \%$.

Los tiempos óptimos de compactación para obtener distintos niveles de Da fueron 0, 24, 50 y $200 \mathrm{~h}$, lo que permitió definir los tratamientos T0, T3, T2 y T1, respectivamente. Con posterioridad a las $200 \mathrm{~h}$ el contenido de agua se estabilizó, alcanzando un valor correspondiente a "capacidad de campo" in situ (Warrick, 2002), de aproximadamente un $16 \%$ en base a masa de suelo seco. El retraso en alcanzar el estado de equilibrio es lógico si se considera la discontinuidad del sistema poroso como consecuencia de la remoción del suelo, además de la presencia del nivel freático cercano a la superficie (Hillel, 1998). Esto permitió que la relación entre el contenido de agua con que se pasó el rodillo y la Da obtenida, presentara el comportamiento esperado, típico de un ajuste parabólico (Figura 3).

El implemento con el cual se ejerce la presión adicional al suelo tiene una influencia en profundidad que depende de su propia masa, además de la superficie en la cual esté en contacto con el suelo (Hartge, 2000; Horn et al., 2007). La disipación de cargas en profundidad explica el menor coeficiente de curvatura y la menor pendiente de la curva de $20-30 \mathrm{~cm}$; asimismo, los valores de Da alcanzados no logran diferenciar claramente los tratamientos, lo que sí ocurre entre $0-20 \mathrm{~cm}$ (Cuadro 2).

Los tratamientos tomaron un ordenamiento de parábola invertida (Figura 3), ya que con contenidos de agua crecientes, los puntos de contacto entre las partículas se lubrican, haciendo más eficiente el proceso de pre-compactación (Wysocka et al., 2006). Sin embargo, se llega a un estado en que el contenido de agua es excesivo y el agua queda confinada en el sistema poroso, sin posibilidad de salir a la velocidad de tránsito del rodillo; de esta forma, se generan presiones internas

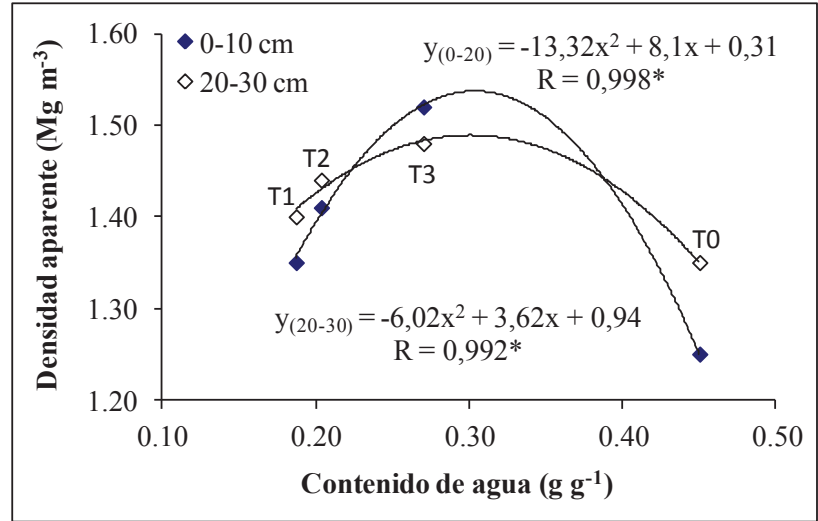

Figura 3: Valores de densidad aparente a dos profundidades en función del contenido de agua con posterioridad al paso del rodillo. Rombo lleno, 0-20 cm; rombo vacío, $20-30 \mathrm{~cm}$. * Significativo al 95\%.

Figure 3: Bulk density values at two depths depending on the water content after roller passage. Full rhombus, 0-20 cm depth; empty rhombus, $20-30 \mathrm{~cm}$ depth. * Significant at 95\%.

Cuadro 2: Densidad aparente (Da, promedio \pm DS) máxima alcanzada luego del paso de un rodillo compactador. $\triangle \mathrm{Da}$ corresponde a la diferencia entre la Da de 0-20 y la de 20-30 cm.

Table 2: Maximum bulk density (Da, average \pm SD) achieved after the passage of a compactor roller. $\Delta$ Da correspond to the difference among Da measured at 0-20 cm and 20-30 cm.

\begin{tabular}{|c|c|c|c|}
\hline \multirow{2}{*}{ Tratamiento } & \multicolumn{2}{|c|}{$\mathrm{Da}\left(\mathrm{Mg} \mathrm{m}^{-3}\right)$} & \multirow{2}{*}{$\frac{\Delta \mathrm{Da}}{\left(\mathrm{Mg} \mathrm{m}^{-3}\right)}$} \\
\hline & $0-20 \mathrm{~cm}$ & $20-30 \mathrm{~cm}$ & \\
\hline T0 & $1,25( \pm 0,09)$ a & $1,35( \pm 0,05)$ a & 0,10 \\
\hline $\mathrm{T} 1$ & $1,35( \pm 0,02) \quad b$ & $1,40( \pm 0,05) \mathrm{a} \mathrm{b}$ & 0,05 \\
\hline $\mathrm{T} 2$ & $1,41( \pm 0,07) \mathrm{b}$ & $1,44( \pm 0,09) \mathrm{a} b$ & 0,03 \\
\hline $\mathrm{T} 3$ & $1,52( \pm 0,05) \quad c$ & $1,48( \pm 0,04) \quad b$ & $-0,04$ \\
\hline
\end{tabular}

Letras distintas denotan diferencias significativas entre tratamientos $(\alpha \leq 0,05)$

en los poros del suelo, lo que ayuda a disipar la carga, disminuyendo el efecto compactador (Karube y Kawai, 2001). Esta es la situación que se asumió para el tratamiento $\mathrm{T} 0$, donde todo el sistema poroso se encuentra lleno de agua al inicio de la curva de agotamiento (Figura 2). Los distintos tratamientos se ordenaron en orden creciente de contenido de agua, aunque el valor final de Da en T3 se logró tanto por efecto lubricante del agua entre las partículas como por exceso de ésta, generándose cargas neumáticas que evitaron alcanzar niveles mayores de Da (Horn, 2003).

En todos los tratamientos, a excepción del T3, aumentó la Da en profundidad (Cuadro 2). Según Ellies y Dörner (1999), los suelos arenosos secos poseen una 
baja transmisión de tensiones en profundidad, lo que se debe a una transferencia energética producida por el roce entre las partículas; así, las tensiones se concentran en superficie. Sin embargo, es necesario considerar que los materiales sueltos poseen un alto factor de concentración, favoreciendo la transmisión de cargas en profundidad (Horn, 1988; Ellies et al., 2000). En la Figura 2 se observa que el rápido secado del material suelo ( 0 a $50 \mathrm{~h}$ posterior al riego) determinó un aumento del roce, obteniendo los menores valores de Da en los tratamientos T1 y T2, pero con una buena transmisión en profundidad, dada su condición suelta. Por su parte, el tratamiento T3, con un alto contenido de agua al momento de pasar el rodillo compactador, tuvo un efecto contrario al señalado por Ellies y Dörner (1999), lo que se pudo deber a la velocidad de paso del rodillo, que generó cargas neumáticas en superficie, previniendo la transmisión de éstas en profundidad (Peng et al., 2004).

Al efectuar la prueba de Próctor en laboratorio, para el mismo suelo con y sin tamizaje ( $2 \mathrm{~mm}$ ), se aprecia un comportamiento similar del suelo evaluado a nivel de campo (Figura 4).

Existe un comportamiento contrastante entre las condiciones de medición (material completo v/s tamizado a $2 \mathrm{~mm}$ ), presentándose diferencias en los máximos encontrados. En la prueba realizada al material tamizado, se encontraron puntos de la curva que sobrepasaron valores de Da de 1,6 $\mathrm{Mg} \mathrm{m}^{-3}$, además se representó una mayor concavidad de la parábola invertida (tanto en la curvatura, como en la pendiente estabilizada, respectivamente, 24,00 y 6,97 en el material tamizado, vs. 18,00 y 5,72 en el no tamizado). La explicación para

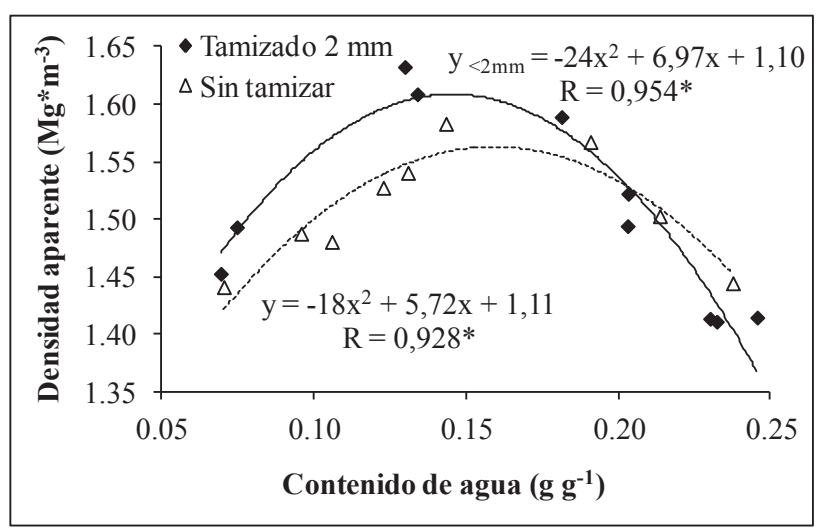

Figura 4: Test de Próctor del material suelo repuesto posterior a la extracción de arena. Rombo lleno, material tamizado a $2 \mathrm{~mm}$; triángulo vacío, material sin tamizar. * Significativo al $95 \%$.

Figure 4: Proctor test of replacement soil material after sand extraction. Full rhombus, 2 mm sieved material; empty triangle, non-sieved material. * Significant at 95\%. esto se encuentra en que el suelo no tamizado presenta material grueso agregado por sílice y/o carbonato, que genera un efecto amortiguador de las cargas aplicadas al suelo (Kézdi, 1980); además, este material agregado posee una humectación más lenta, lo que provoca núcleos de fricción mayor que dificultan la transmisión de las tensiones (Ellies y Dörner, 1999).

En campo, estos materiales están repartidos al azar en todo el volumen del suelo disturbado por la extracción de áridos, lo que explicaría en parte la menor Da alcanzada en estas condiciones. Un aspecto notable es que las curvas de la Figura 4 poseen casi el mismo intercepto (1,10 y 1,11 $\left.\mathrm{Mg} \mathrm{m}^{-3}\right)$, los cuales son menores incluso a la Da obtenida en el T0, lo que indica que una pre-compactación sobre el material en estado seco no hubiese sido efectiva en alcanzar una Da que asegurase una adecuada capacidad de soporte (Schäffer et al., 2010).

Al comparar las figuras 3 y 4 , se observa que la pendiente estabilizada de la parábola es mayor en el Próctor de campo en superficie respecto al Test normado $(8,10$ vs. 6,97$)$, aunque no ocurre lo mismo en profundidad. Esto resulta en un intercepto (contenido de agua $=0)$, menor en la prueba de campo $\left(0,3 \mathrm{Mg} \mathrm{m}^{-3}\right)$. Considerando que en terreno, con un nivel freático cercano a la superficie, es difícil lograr un estado "seco al aire" del suelo, el comportamiento de la prueba de campo es lógico, por cuanto con rangos de contenido de agua mayores se obtienen valores de Da menores respecto al Test de Próctor. Además, esta prueba aplica una mayor energía a través de la carga dinámica aplicada, respecto a la carga dinámica ejercida sólo por el tránsito del rodillo en terreno (Das, 1997).

\section{Espacio poroso}

Como era de esperar, la distribución de tamaño de poros respondió al tratamiento de pre-compactación, disminuyendo los poros gruesos a medida que aumentó la efectividad de la labor, los cuales colapsaron a tamaños de poros de retención de agua y menores. Los resultados se presentan en el Cuadro 3, donde se observa que las dos profundidades evaluadas presentaron un comportamiento similar entre sí.

Como producto de la pre-compactación de los suelos disturbados, se logró obtener relaciones de porosidad muy características para los tratamientos más compactados (T2 y T3) y los menos compactados (T0 y T1). Con relación a los poros de drenaje rápido (PDR), los tratamientos menos compactados alcanzaron valores mayores y diferentes estadísticamente al T2 y T3, condición que los predispone a un flujo de agua muy rápido en el perfil.

Pagliai y Vignozzi (2002) consideran que suelos de mineralogía cristalina con una proporción de poros mayores a $50 \mu \mathrm{m}$ (PDR) entre 10 y $25 \%$ corresponden a suelos moderadamente porosos, lo que en este caso 
Cuadro 3: Distribución de los tamaños de poros del suelo, acorde a distintos niveles de pre-compactación.

Table 3: Pore size distribution, according to different levels of pre-compaction.

\begin{tabular}{cccc}
\hline Tratamiento & PDR & PAU & PAI \\
\hline \multicolumn{5}{c}{ Profundidad 0-20 cm } \\
\hline T0 & $24,6( \pm 4,2)$ b & $17,3( \pm 2,9)$ a \\
T1 & $22,9( \pm 2,1)$ b & $18,7( \pm 2,2)$ a b & $6,7( \pm 0,2)$ a \\
T2 & $18,8( \pm 4,3)$ a & $18,7( \pm 2,6)$ a b & $7,0( \pm 0,2)$ b \\
T3 & $15,2( \pm 3,0)$ a & $22,7( \pm 4,0)$ b & $7,9( \pm 0,3) \quad$ c \\
\hline \multicolumn{5}{c}{ d } \\
\hline T0 & $22,2( \pm 4,7)$ b & $16,7( \pm 3,6)$ a & $6,5( \pm 0,3)$ a \\
T1 & $22,9( \pm 5,1)$ b & $17,7( \pm 2,4)$ a b & $7,1( \pm 0,1)$ b \\
T2 & $16,2( \pm 5,3)$ a & $19,3( \pm 3,0)$ b & $7,3( \pm 0,3) \quad$ c \\
T3 & $13,4( \pm 4,4)$ a & $23,1( \pm 1,2) \quad$ c & $7,8( \pm 0,2) \quad$ d \\
\hline
\end{tabular}

A una misma profundidad, letras distintas denotan diferencias significativas entre tratamientos $(\alpha \leq 0,05)$. PDR: poros de drenaje rápido, PAU: poros de agua útil, PAI: poros de agua no útil.

se cumpliría en todos los tratamientos, asegurando una porosidad de aireación mínima para los requerimientos de los distintos cultivos. A su vez, Horn y Fleige (2009) señalan que en un amplio rango de suelos basta un $8 \%$ de poros gruesos para mantener las condiciones adecuadas para los cultivos, toda vez que dicha porosidad sea lo suficientemente continua para mantener la funcionalidad física del suelo.

Los poros de drenaje lento (PDL, 10-50 $\mu \mathrm{m}$, datos no incluidos) variaron entre 8 y $9 \%$, sin diferencias estadísticas significativas entre los distintos tratamientos. En los tratamientos T3 y T2 se encontraron los mayores porcentajes de poros de agua útil (PAU, 0,2-10 $\mu \mathrm{m}$, retención entre -33 y -1500 kPa), siendo éstos diferentes estadísticamente entre sí entre los 20 y $30 \mathrm{~cm}$ de profundidad. Esta condición determina una mayor disponibilidad de agua para los cultivos que se establezcan; aún así, todos los tratamientos presentaron valores de PAU en el rango ideal a bueno, de acuerdo a Reynolds et al. (2009).

Los poros de agua no disponible (PAI), presentaron diferencias estadísticas entre tratamientos, aunque este parámetro depende mayoritariamente de la textura que presenta el suelo. Al tratarse del mismo suelo, estos resultados estarían mostrando dependencia entre el nivel de densificación y el contenido de agua al momento de pre-compactar, resultado del acomodo entre los distintos tamaños de partículas presentes (Hartge, 2000). Sin embargo, la diferencia de PAI entre el valor menor $(6,5 \%$, T0 entre 20 y $30 \mathrm{~cm})$ y el valor mayor $(7,9 \%$, T3 entre 0 y $20 \mathrm{~cm})$ no es determinante en términos de manejo (Rawls et al., 2003).

Otro parámetro que permite analizar los vacíos del suelo es la relación de vacío o número poroso $\left(e, \mathrm{~m}^{-3} \mathrm{de}\right.$ poros $\mathrm{m}^{-3}$ de sólidos), valor que refleja de mejor forma los cambios en el volumen de poros de un suelo producto de su manejo (Hartge y Horn, 2009). El Cuadro 4 presenta el valor $e$ de los distintos tratamientos.

Los valores de $e$ se encuentran dentro del rango de 0,58-1,22 señalado para suelos de clases texturales gruesas (Scheffer y Schachtschabel, 1992), donde valores superiores a 1,00 indican una porosidad superior al $50 \%$. En la profundidad de $0-20 \mathrm{~cm}$ se presentaron diferencias significativas entre los tratamientos, siendo los tratamientos que alcanzaron una mayor consolidación los que obtuvieron un menor número poroso. Para la profundidad de $20-30 \mathrm{~cm}$, se mantiene la tendencia, aunque, al igual que con la Da (Cuadro 2), se pierde el

Cuadro 4: Número poroso ( $e$, promedio $\pm \mathrm{DS}$ ) para los distintos tratamientos. $\Delta e$ corresponde a la diferencia entre los valores de e entre 0-20 y 20-30 cm.

Table 4: Void ratio ( $e$, average $\pm \mathrm{SD}$ ) for different treatments. $\Delta e$ correspond to the difference between e values measured at $0-20 \mathrm{~cm}$ and $20-30 \mathrm{~cm}$

\begin{tabular}{|c|c|c|c|}
\hline \multirow[t]{2}{*}{ Tratamiento } & \multicolumn{2}{|c|}{$e\left(\mathrm{~m}^{-3}\right.$ poros $\mathrm{m}^{-3}$ de sólidos $)$} & \multirow[t]{2}{*}{$\Delta e$} \\
\hline & $0-20 \mathrm{~cm}$ & $20-30 \mathrm{~cm}$ & \\
\hline T0 & $1,146( \pm 0,18)$ a & $0,990( \pm 0,08) \mathrm{a}$ & 0,156 \\
\hline $\mathrm{T} 1$ & $0,981( \pm 0,03) b$ & $0,906( \pm 0,07) b$ & 0,075 \\
\hline $\mathrm{T} 2$ & $0,899( \pm 0,10) b$ & $0,861( \pm 0,11)$ bc & 0,038 \\
\hline T3 & $0,761( \pm 0,06) \quad \mathrm{c}$ & $0,807( \pm 0,05) \quad c$ & $-0,046$ \\
\hline
\end{tabular}

Letras distintas denotan diferencias significativas entre tratamientos $(\alpha \leq 0,05)$. 
efecto de transmisión de cargas (reflejado por la diferencia entre profundidades, $\Delta$ ) al aumentar la pre-compactación, debido a la concentración de la carga en la superficie.

La distinta distribución de tamaño de poros se debiese reflejar en el comportamiento hidráulico del suelo, principalmente con los procesos relacionados con el drenaje y almacenamiento de agua al momento del riego o al producirse un evento de lluvia. Para evaluar este fenómeno, se midió el contenido de agua del suelo 24 $\mathrm{h}$ después de una lluvia natural de aproximadamente $20 \mathrm{~mm}$ (Cuadro 5).

Efectivamente, se presentó un mayor contenido de agua en los tratamientos T3 y T2, es decir, aquellos con mayor pre-compactación. Estos resultados confirman la existencia de un mayor porcentaje de poros de almacenamiento y retención de agua en estos tratamientos, respecto de los tratamientos con suelo más suelto (T0 y T1).

La relación entre Da y contenido de agua es del tipo exponencial, con un ajuste significativo al 95\%, repre-

Cuadro 5: Agua almacenada en el suelo $(0-20 \mathrm{~cm}) 24 \mathrm{~h}$ después de una lluvia de $20 \mathrm{~mm}$ para cada tratamiento de precompactación. Promedio \pm DS.

Table 5: Stored water in the soil $(0-20 \mathrm{~cm}) 24 \mathrm{~h}$ after a 20 $\mathrm{mm}$ rainfall for each treatment of pre-compaction. Average \pm SD.

\begin{tabular}{ccc}
\hline Tratamiento & Contenido de agua & Da \\
\hline & $\left(\mathrm{g} \mathrm{g}^{-1}\right)$ & $\left(\mathrm{Mg} \mathrm{m}^{-3}\right)$ \\
\hline T0 & $6,86( \pm 2,50) \mathrm{a}$ & 1,25 \\
T1 & $7,23( \pm 2,13) \mathrm{a} \mathrm{b}$ & 1,35 \\
T2 & $10,37( \pm 5,62) \quad$ b & 1,41 \\
T3 & $17,34( \pm 3,23) \quad$ c & 1,52 \\
\hline
\end{tabular}

Letras distintas denotan diferencias significativas entre tratamientos $(\alpha \leq 0,05)$. sentado por la ecuación y = 1,17 $\mathrm{e}^{0,0154 x}$. Esta relación es característica para cada tipo de suelo, el que presentará un máximo almacenamiento de agua determinado por la estructura y la textura, las cuales se manifiestan en la porosidad total (Warrick, 2002).

\section{Resistencia a la penetración}

Uno de los efectos que genera la pre-compactación de un suelo, además del aumento en la Da y la modificación de la distribución de tamaño de poros, es el aumento en la resistencia a la penetración (RP). La medición de este parámetro permite una caracterización de los cambios tanto morfológicos (consistencia) como mecánicos experimentados por el suelo en cada uno de los tratamientos. Los resultados para las mediciones de RP en profundidad se presentan en el Cuadro 6, mediante la evaluación realizada con el contenido de agua alcanzado $24 \mathrm{~h}$ después de una lluvia artificial de $20 \mathrm{~mm}$, de manera de estandarizar el estado energético del agua del suelo.

Para todos los tratamientos, se observa un incremento de la RP hasta una profundidad de $25 \mathrm{~cm}$. El tratamiento T0 (testigo) posee los menores valores, aunque sin diferencias estadísticas en relación al T1.

En superficie $(5 \mathrm{~cm})$, las diferencias de RP no se hacen evidentes en relación al grado de compactación de los tratamientos, posiblemente por la soltura del suelo generada por la labores de siembra y control de malezas. Solo el tratamiento T3 muestra diferencias significativas con el resto de los tratamientos. Tal como lo señala Hartge (2000), al aplicar una carga alta, las tensiones se concentran en superficie, por lo que se registran los mayores valores de RP, disipándose rápidamente en profundidad; esta situación ya se había discutido en torno a los valores de Da (Cuadro 2).

Bajo los $25 \mathrm{~cm}$, las relaciones entre los tratamientos desaparecen y no siguen una tendencia similar a las primeras profundidades. Este comportamiento se debe a que la presión ejercida por el tratamiento no influye

Cuadro 6: Resistencia a la penetración a distintas profundidades de suelo para los tratamientos de pre-compactación.

Table 6: Penetration resistance at different soil depths for the treatments of pre-compaction.

\begin{tabular}{ccccccc}
\hline Tratamiento & \multicolumn{5}{c}{ Resistencia a la penetración (kPa) } \\
\hline & 5 & 15 & 25 & 35 & 55 & 75 \\
T0 & $47 \mathrm{a}$ & $178 \mathrm{a}$ & $210 \mathrm{a}$ & $198 \mathrm{a}$ & $184 \mathrm{a}$ & $156 \mathrm{a}$ \\
T1 & $49 \mathrm{a}$ & $201 \mathrm{a} \mathrm{b}$ & $224 \mathrm{a} \mathrm{b}$ & $178 \mathrm{a}$ & $143 \mathrm{~b}$ & $137 \mathrm{a}$ \\
T2 & $63 \mathrm{a}$ & $225 \mathrm{~b}$ & $287 \quad \mathrm{c}$ & $213 \mathrm{a}$ & $184 \mathrm{a}$ & $161 \mathrm{a}$ \\
T3 & $99 \mathrm{~b}$ & $282 \mathrm{c}$ & $274 \mathrm{~b} \mathrm{c}$ & $208 \mathrm{a}$ & $128 \mathrm{~b}$ & $101 \mathrm{~b}$ \\
\hline
\end{tabular}

Letras distintas en cada columna indican diferencias estadísticas significativas entre tratamientos $(\alpha \leq 0,05)$. 
a mayor profundidad, debido a que la clase textural del suelo y el nivel freático actúan como amortiguadores de las cargas externas (Ellies y Dörner, 1999). Para el caso del testigo (T0) la generación de resistencia en profundidad se debe a un proceso de asentamiento natural (Hartge, 1988), en tanto el T1 no se habría visto afectado por el paso del rodillo bajo los $35 \mathrm{~cm}$ de profundidad.

Si bien el T2 posee valores de resistencia superiores al valor crítico de $200 \mathrm{kPa}$ señalado en la literatura (Taylor y Brar, 1991), como se mostrará más adelante, es el que presentó mayor rendimiento en materia seca (Cuadro 8), por lo que la redistribución de poros de aire a poros de retención de agua (Cuadro 3) sería más importante que el aumento de la resistencia mecánica en un suelo franco arenoso. La explicación está dada porque si bien los materiales arenosos tienen una alta resistencia por el mayor roce entre partículas (Ashburner y Sims, 1984), el espacio poroso entre las partículas de arena permite mantener una matriz rígida con el espacio suficiente para el crecimiento de raíces (Mitchell, 1993).

\section{Comportamiento hidráulico del suelo}

La aplicación de cargas normales con distintos contenidos de agua en el suelo genera diferencias en el ordenamiento de las partículas, influyendo a nivel de las conexiones entre los poros y disminuyendo los diámetros de éstos, debido a que las partículas de suelos están más cercanas entre sí. La Figura 5 presenta los resultados de infiltración acumulada, la cual siguió una tendencia esperable en función de los tratamientos. El Cuadro 7, en tanto, incluye valores de conductividad hidráulica saturada (Ks), considerada como la VI al minuto 1 , de acuerdo a una aproximación de Kostiakov (Gurovich, 1985).

De acuerdo a la Figura 5 y al Cuadro 7, todos los tratamientos presentan un alto riesgo de lixiviación de nutrientes y contaminantes, ya que de acuerdo a Casanova et al. (2008) poseen una Ks muy alta $(>36 \mathrm{~cm}$ $\mathrm{h}^{-1}$ ), por lo que se justificaría plenamente la labor de pre-compactación, la cual no sólo mejoraría la traficabilidad del suelo, sino que mejoraría su función ambiental (Powlson et al., 2011). Sin embargo, al calcular la infiltración estabilizada (minuto 60 en adelante) y realizar un ajuste lineal de los valores, las pendientes de las rectas de la Figura 5 corresponden a una tasa de infiltración de 7,3 $\mathrm{cm} \mathrm{h}^{-1}$ para T3 y 13,1 $\mathrm{cm} \mathrm{h}^{-1}$ para T0 (Cuadro 7), lo que las categoriza como moderadamente rápida y rápida, respectivamente, con diferencias estadísticas significativas más claras respecto a los resultados de Ks.

El hecho de no alcanzar valores de Da similares a los del suelo original, que alcanzan en promedio 1,6 - 1,7 $\mathrm{Mg} \mathrm{m}^{-3}$ (Rojas, 2007), se explica por la disipación de la
Cuadro 7: Valores de conductividad hidráulica y de pendiente estabilizada de la curva de infiltración para cada tratamiento.

Table 7: Hydraulic conductivity values and stabilized slope of the infiltration curve for each treatment.

\begin{tabular}{ccc}
\hline Tratamiento & $\begin{array}{c}\text { Conductividad } \\
\text { hidráulica }\end{array}$ & $\begin{array}{c}\text { Pendiente estabilizada } \\
\text { de curva de infiltración }\end{array}$ \\
\hline & $\left(\mathrm{cm} \mathrm{h}^{-1}\right)$ & $\left(\mathrm{cm} \mathrm{h}^{-1}\right)$ \\
T0 & $47,7( \pm 6,38)$ b & $13,1 \mathrm{a}$ \\
T1 & $45,9( \pm 7,15)$ a b & $12,1 \mathrm{a} \mathrm{b}$ \\
T2 & $38,3( \pm 1,75)$ a & 11,2 b \\
T3 & $37,5( \pm 6,31)$ a b & $7,3 \quad$ c \\
\hline
\end{tabular}

Letras distintas en cada columna indican diferencias estadísticas significativas entre tratamientos $(\alpha \leq 0,05)$.

Cuadro 8: Rendimiento promedio de arvejas para cada tratamiento de pre-compactación.

Table 8: Average yield of peas for each treatment of precompaction.

\begin{tabular}{cc}
\hline \multirow{2}{*}{ Tratamientos } & \multicolumn{1}{c}{ Materia seca } \\
\cline { 2 - 2 } & \multicolumn{1}{c}{$\left(\mathrm{Mg} \mathrm{ha}^{-1}\right)$} \\
\hline T0 & $0,75( \pm 0,15) \mathrm{a}$ \\
T1 & $0,91( \pm 0,33) \mathrm{a} \mathrm{b}$ \\
T2 & $1,10( \pm 0,42) \quad \mathrm{b}$ \\
T3 & $0,82( \pm 0,18) \mathrm{a}$ \\
\hline
\end{tabular}

Letras distintas denotan diferencias significativas entre tratamientos $(\alpha \leq 0,05)$.

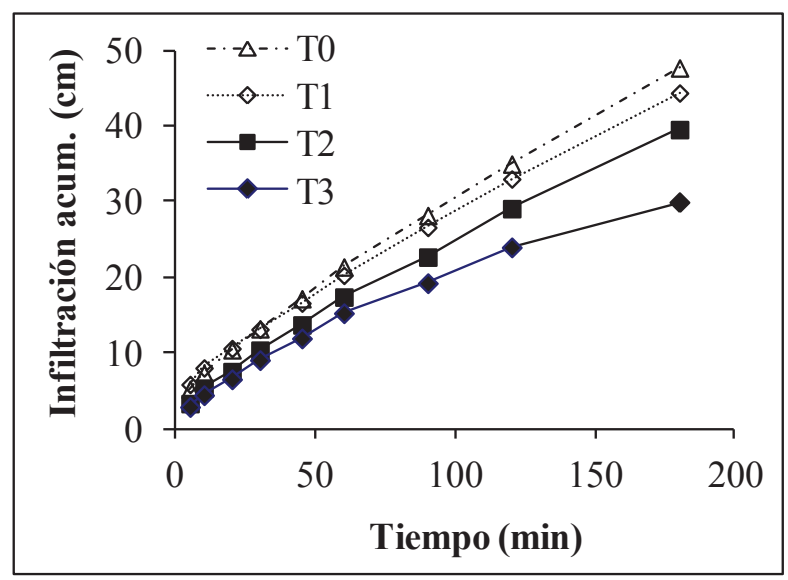

Figura 5: Curvas de infiltración acumulada para los tratamientos de pre-compactación.

Figure 5: Cumulative infiltration curves for pre-compaction treatments. 
carga aplicada y la poca tensión ejercida por el rodillo compactador. Esto se traduce en que en los primeros minutos de la prueba de infiltración no se generan diferencias entre tratamientos, aunque con el tiempo sí, lo que se corrobora con la prueba de la pendiente estabilizada.

\section{Rendimiento de materia seca del cultivo}

La producción de materia seca fue medida con plantas extraídas completamente al final del ensayo, al momento de alcanzar las arvejas el estado de vaina comercial. Este parámetro se utilizó para determinar qué tratamiento ofreció un mejor ambiente edáfico para el crecimiento de los vegetales, pensando en realizar un cultivo de cobertura que contribuya a la estructuración de suelo en el corto plazo (Abiven et al., 2009). Dado su rápido crecimiento, se eligió la especie $P$. sativum como planta indicadora, aunque otros autores han utilizado praderas (poáceas), alcanzando resultados comparables con esta investigación (Ellies et al., 1992; Medvedev, 2004). Los resultados se presentan en el Cuadro 8.

En la expresión gráfica de estos resultados (Figura 6), se observa una tendencia a disminuir el rendimiento en términos de fitomasa para ambos extremos de precompactación. El T0 presenta una soltura extrema, baja disponibilidad de agua para el cultivo y un exceso de aire en el suelo (Hartge y Ellies, 1999); por otro lado, el T3 posee la mayor resistencia a la penetración, un espacio poroso reducido y una continuidad menor del sistema poroso, generando obstáculos para el crecimiento de las raíces (Ellies et al., 1992).

Otros autores (Lhotský et al., 1991; Lindstrom y Voorhees, 1994) han encontrado el mismo comportamiento de parábola invertida para otros tipos de cultivos, aunque con rendimientos mayores debido a que

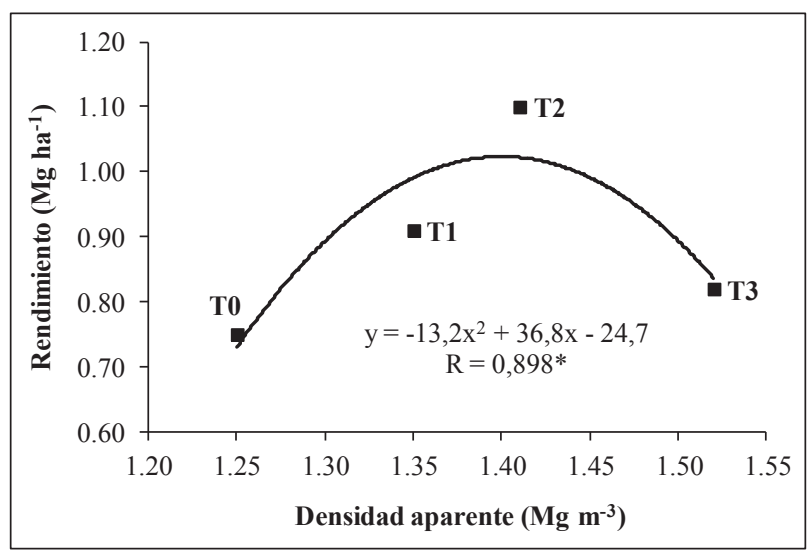

Figura 6: Materia seca de arvejas, para distintos niveles de pre-compactación. * Significativo al 95\%.

Figure 6: Dry matter of peas, for different levels of pre-compaction. * Significant at $95 \%$. trabajaron en suelos no disturbados, disponiendo de distintos niveles de compactación debido al manejo. Para el caso de la cebada, el rendimiento de grano puede ser mayor a $4 \mathrm{Mg} \mathrm{ha}^{-1}$ en un suelo areno francoso (Petelkau y Siedel, 1986), aunque durante una estación seca en suelos franco-finos, el rendimiento puede ser menor a $1 \mathrm{Mg} \mathrm{ha}^{-1}$ con valores de Da de $1,2 \mathrm{Mg} \mathrm{m}^{-3}$ (Shipilov, 1982, citado por Lipiec y Simota, 1994).

El máximo de la curva expresa la relación óptima en la proporción de aire-agua-sólidos del suelo, para el crecimiento del cultivo empleado, obtenido a valores de pre-compactación representados por el T2. Para suelos franco arenosos, la pérdida de producción de biomasa por aumento de la Da obedece al ordenamiento de partículas, que provoca un aumento en la tortuosidad y una disminución de la porosidad gruesa, siendo estos factores más relevantes que el aumento de la resistencia mecánica (Lindstrom y Voorhees, 1994).

La pre-compactación de suelos, si bien ya había sido utilizada como técnica de recultivación o con objetivos de almacenamiento de agua (Ellies y Álvarez, 1985; Hartge, 2000), no había sido utilizada post-extracción de áridos en suelos de texturas gruesas. Si bien no se alcanzaron los niveles de Da de la pedogénesis natural, se logra la capacidad de soporte y la redistribución del sistema poroso que permiten la funcionalidad del suelo en la conducción de agua y aire, permitiendo la producción agrícola al primer año de reposición del suelo. Sin embargo, aún es necesario investigar la dinámica del carbono orgánico y la evolución de la estructura a través del tiempo en sistemas de este tipo.

\section{CONCLUSIONES}

Es posible realizar la prueba de Próctor de un suelo en condiciones de campo con el tránsito de un rodillo compactador, utilizando distintos contenidos de agua del suelo. Para alcanzar valores de densidad aparente (Da) cercanos a la condición inicial o natural del suelo, es necesario utilizar cargas o tensiones de mayor magnitud a las utilizadas en esta investigación.

El efecto inmediato de la pre-compactación fue la disminución de poros gruesos y un consecuente aumento de la cantidad de poros de retención de agua. Se generaron diferencias en los sistemas porosos con los tratamientos de pre-compactación, sin llegar a poner en riesgo el suministro gaseoso necesario para las plantas, efectuado por los poros de aireación.

La Da óptima del suelo franco arenoso disturbado por la extracción de áridos fluctuó entre 1,4 y 1,5 Mg $\mathrm{m}^{-3}$, lo que aseguró una capacidad de soporte adecuada y una relación agua/aire óptima para el desarrollo de un cultivo de arveja. En relación a la resistencia a la penetración, ésta aumentó a medida que los poros gruesos fueron disminuyendo, sobrepasando ligeramente un valor crítico de $200 \mathrm{kPa}$. Por su parte, el com- 
portamiento hidráulico del suelo presentó diferencias significativas en el tramo analizado con las pendientes estabilizadas, posterior a los 60 minutos del transcurso de la prueba de infiltración.

Las condiciones físico-mecánicas del suelo se vieron mejoradas en los tratamientos que obtuvieron una mayor $\mathrm{Da}$, con valores óptimos alcanzados por el tratamiento T2 (condición intermedia de contenido de agua al momento de la pre-compactación), lo que fue corroborado por los rendimientos de materia seca del cultivo de arveja.

\section{REFERENCIAS}

ABIVEN, S., MENASSERI, S., CHENU, C. 2009. The effects of organic inputs over time on soil aggregate stability - A literature analysis. Soil Biology \& Biochemistry 41 (1): 1-12.

ALLEN, R.G., PEREIRA, L.S., RAES, D., SMITH, M. 2006. Evapotranspiración del cultivo. Guías para la determinación de los requerimientos de agua de los cultivos. Estudio FAO Riego y Drenaje 56. Roma, Italia.

AMERICAN SOCIETY FOR TESTING AND MATERIALS (ASTM). 1995. Annual Book of ASTM Standards. Vol 04.08, Philadelphia, USA.

ASHBURNER, J., SIMS, B. 1984. Elementos de Diseño del Tractor y Herramientas de Labranza. Instituto Interamericano de Cooperación para la Agricultura (IICA). San José, Costa Rica.

CASANOVA, M., SEGUEL, O., HABERLAND, J., KREMER, C. 2008. Propiedades físicas: Indicadores de calidad y salud del suelo. Antumapu-Revista de extensión y comunicación Agropecuaria y Medio Ambiental 6(1-2): 32-34.

CIREN, 1996. Descripción de Suelos: Materiales y símbolos. Estudio Agrológico Región Metropolitana. Centro de Información de Recursos Naturales. Publicación № 115. Santiago, Chile.

DANE, J.H., HOPMANS, J.W. 2002. Water retention and storage. In: Dane, J.H., Topp, G.C. (Eds.), Methods of Soil analysis. Part 4. Physical Methods. Soil Science Society of America, Book Series № 5, Wisconsin, pp. 671-720.

DAS, B.M. 1997. Soil Mechanics Laboratory Manual. Fifth Edition. Engineering Press, Austin, USA.

ELLIES, A., ÁLVAREZ, I. 1985. Economía del agua en el período estival para pradera, maíz forrajero y col forrajero en un Typic Dystrandept de Valdivia. Agro Sur 13(1): 39-50.

ELLIES, A., RAMÍREZ, C., MACDONALD, R., FIGUEROA, H., GAYOSO, J. 1992. Efecto de la compactación de suelos volcánicos sobre el crecimiento de Lolium multiflorum y Lolium pratense. Agricultura Técnica 52(4): 450-455.

ELLIES, A., DÖRNER, J. 1999. Distribución de tensiones en un suelo Palehumult ejercida con el tráfico de la maquinaria agrícola. Agro Sur 27(2): 1-9.

ELLIES, A., HORN, R., SMITH, R. 2000. Effect of management of a volcanic ash soil on structural properties. International Agrophysics 14(4): 377-384.

FLINT, A.L., FLINT, L.E. 2002. Particle density. In: Dane, J.H., Topp, G.C. (Eds.), Methods of Soil Analysis. Part 4. Physical Methods. Soil Science Society of America, Book Series № 5, Wisconsin, pp. 229-240.

GROSSMAN, R.B., REINSCH, T.G. 2002. Bulk density and linear extensibility. In: Dane, J.H., Topp, G.C. (Eds.), Methods of Soil analysis. Part 4. Physical Methods. Soil Science Society of America, Book Series № 5, Wisconsin, pp. 201228.

GUROVICH, L. 1985. Fundamentos y diseño de sistemas de riego. Instituto Interamericano de Cooperación para la Agricultura (IICA). San José, Costa Rica.

HARTGE, K. H. 1988. The reference base for compaction state of soils. In: Drescher, J., Horn, R., de Boodt, M. (Eds.), Impact of water and external forces on soil structure. Catena (Supplement 11), Reiskirchen, pp. 73-77.

HARTGE, K. H., ELLIES, A. 1999. El rol de la física de suelos en la producción agrícola. Agro Sur 27(1): 43-56.

HARTGE, K.H. 2000. The effect of soil deformation on physical soil properties. A discourse on the common background. In: Horn, R., Van den Akker, J.J.H., Arvidson, J. (Eds.), Subsoil Compaction. Distribution, processes and consequences. Advances in Geoecology 32. Catena Verlag, Reiskirchen, pp. 32-43.

HARTGE, K. H., HORN, R. 2009. Die physikalische Untersuchung von Böden. Praxis Messmethoden Auswertung. 4 vollst. Überarbeitete Auflage. Schweizerbart, Vorlage, Stuttgart, Germany.

HILLEL, D. 1998. Environmental soil physics. Academic Press. San Diego, USA.

HORN, R. 1988. Compressibility of arable land. In: Drescher, J., Horn, R., de Boodt, M. (Eds.), Impact of water and external forces on soil structure. Catena (Supplement 11), Reiskirchen, pp. 53-71.

HORN, R. 2003. Stress-strain effects in structured unsaturated soils on coupled mechanical and hydraulic processes. Geoderma 116(12): 77-88.

HORN, R., HARTGE, K.H., BACHMANN, J., KIRKHAM, M.B. 2007. Mechanical stresses in soils assessed from bulkdensity and penetration-resistance data sets. Soil Science Society of America Journal 71(5): 1455-1459.

HORN, R., FLEIGE, H. 2009. Risk assessment of subsoil compaction for arable soils in Northwest Germany at farm 
scale. Soil \& Tillage Research 102(2): 201-208.

KARUBE, D., KAWAI, K. 2001. The role of pore water in the mechanical behaviour of unsaturated soils. Geotechnical and Geology Engineering 19: 211-241.

KÉZDI, A. 1980. Handbook of Soil Mechanics. Vol. 2. Soil testing. Elsevier, Amsterdam.

KOOLEN, A.J., KUIPERS, H. 1983. Agricultural Soil Mechanics. Advanced Series in Agricultural Sciences 13. SpringerVerlag, Berlin.

LHOTSKÝ, J., BERAN, P., PARIS, P., VALIGURSKÁ, L. 1991. Degradation of soil by increasing compression. Soil and Tillage Research 19(2-3): 287-295.

LINDSTROM, M.J., VOORHEES, W.B. 1994. Responses of temperate crops in North America to soil compaction. In: Soane, B.D., van Ouwerkerk, C. (Eds.), Soil Compaction in Crop Production. Developments in Agricultural Engineering 11. Elsevier, Amsterdam, pp. 265-286.

LIPIEC, J., SIMOTA, C. 1994. Role of soil and climate factors in influencing crop responses to soil compaction in Central and Eastern Europe. In: Soane, B.D., van Ouwerkerk, C. (Eds.), Soil Compaction in Crop Production. Developments in Agricultural Engineering 11. Elsevier, Amsterdam, pp. 365-390.

LIPIEC, J. 2004. Compaction effect on soil physical properties and root and shoot growth. In: Glińsky, J., Józefaciuk, G, Stahr, K. (Eds.), Soil-plant-atmosphere aeration and environmental problems. Centre of Excellence for Applied Physics in Sustainable Agriculture. Lublin - Stuttgart, pp. 124-133.

MACAYA, C., GALLARDO, M. 2007. Manual de rehabilitación de suelos sometidos a extracción de áridos en zonas agrícolas. Gobierno de Chile, Ministerio de Agricultura, Servicio Agrícola y Ganadero. Santiago, Chile.

MEDVEDEV, V. 2004. Soil compaction as a factor of soil productivity and ecological functions in the Ukraine. In: Lipiec, J., Walczak, R., Józefaciuk, G. (Eds.), Plant growth in relation to soil physical conditions. Centre of Excellence for Applied Physics in Sustainable Agriculture. Lublin, pp. 95-102.

MITCHELL, J.K. 1993. Fundamentals of soil behavior. John Wiley \& Sons, New York.

OLIVARES, A., JOHNSTON, M., CONTRERAS, X. 1998. Régimen Pluviométrico del Secano Interior de la Región Metropolitana. Avances en Producción Animal 23(1-2): 35-43.

PAGLIAI, M., VIGNOZZI, N. 2002. The soil pore systems as an indicator of soil quality. In: Pagliai, M., Jones, R. (Eds.), Sustainable Land Management - Environmental Protection. A Soil Physical Approach. IUSS - UISS - IBU. Reiskirchen, pp. 71-82.
PENG, X.H., HORN, R., ZHANG, B., ZHAO, Q.G. 2004. Mechanisms of vulnerability to compaction of homogenized and recompacted Ultisols. Soil and Tillage Research 76(2): 125-137.

PETELKAU, H., SEIDEL, K. 1986. Workability and trafficability of arable soils as influenced by soil water (in German). Tag. -Ber., Akad. Landwirtsch. Wiss. D.D.R.. Berlin, Germany, 246: 46-54.

POWLSON, D.S., GREGORY, P.J., WHALLEY, W.R., QUINTON, J.N., HOPKINS, D.W., WHITMORE, A.P., HIRSCH, P.R., GOULDING, K.W. 2011. Soil management in relation to sustainable agriculture and ecosystem services. Food Policy 36: S72-S87.

RAWLS, W.J., PACHEPSKY, Y.A., RITCHIE, J.C., SOBECKI, T.M., BLOODWORTH, H. 2003. Effect of soil organic matter carbon on soil water retention. Geoderma 116(1): 61-76.

REYNOLDS, W.D., ELRICK, D.E., YOUNGS, E.G., MOOZEGAR, A., BOOLTINK, H.W.G., BOUMA, J. 2002. Saturated and fieldSaturated water flow parameters. In: Dane, J.H., Topp, G.C. (Eds.), Methods of Soil Analysis. Part 4. Physical Methods. Soil Science Society of America. Book Series № 5, Madison, Wisconsin, pp. 797-878.

REYNOLDS, W.D., DRURY, C.F., TAN, C.S., FOX, C.A., YANG, X.M. 2009. Use of indicators and pore volume-function characteristics to quantify soil physical quality. Geoderma 152 (3-4): 252-263.

ROJAS, I. 2007. Efecto de la aplicación de dos coadyuvantes de riego sobre la apertura del bulbo de mojamiento generado por goteros en un suelo franco arenoso. Memoria Ing. Agr. Universidad de Chile. 43 p.

SCHÄFFER, B., BOIVIN, P., SCHULIN, R. 2010. Compressibility of repacked soil as affected by wetting and drying between uniaxial compression tests. Soil Science Society of America Journal 74(5): 1483-1492.

SCHEFFER, F., SCHACHTSCHABEL, P. 1992. Lehrbuch der Bodenkunde, 13. Aufl. - Enke, Stuttgart.

SILVA, G.I., LIMA, H.V., CAMPANHA, M.M., GILKES, R.J., OLIVEIRA, T.S. 2011. Soil physical quality of Luvisols under agroforestry, natural vegetation and conventional crop management systems in the Brazilian semi-arid region. Geoderma 167-168: 61-70.

TAYLOR, H.M., BRAR, G.S. 1991. Effect of soil compaction on root development. Soil and Tillage Research 19(2-3): 111-119.

WARRICK, A.W. 2002. Soil physics companion. CRC Press, Boca Raton, USA.

WYSOCKA, A., STEPNIEWSKY, W., HORN, R. 2006. Shrinkage properties of three clay materials at different temperatures. International Agrophysics 20(3): 255-260. 